\title{
Is Elective Cancer Surgery Safe During the COVID-19 Pandemic?
}

\author{
${\text { Chenchen } \mathrm{Ji}^{1} \cdot \text { Kaushiki Singh }^{1} \cdot \text { Alison Zoe Luther }}^{1,2}$ (1) $\cdot$ Avi Agrawal $^{1}$
}

Accepted: 8 July 2020/Published online: 6 August 2020

(C) Société Internationale de Chirurgie 2020

\begin{abstract}
Background The COVID-19 pandemic has resulted in a significant decrease in the number of elective cancer operations performed. Cancer patients are felt to be a high-risk group for COVID-19, and therefore, concerns have been raised regarding the safety of operating during this time; however, the potential risk of cancer progression if untreated must also be considered. The aim of this study was therefore to identify the incidence of COVID-19 postoperatively in patients undergoing elective cancer surgery of all types.

Methods Data were collected on all patients who had an elective therapeutic cancer operation in a single large district general hospital, where standard COVID-19 precautions were in place, between 01/02/2020 and 27/4/2020, Follow-up was for a minimum of 2 weeks post-discharge. The primary outcome was the incidence of COVID-19 during the follow-up period.

Results A total of 621 elective cancer surgeries, from a range of specialities, were performed during the study period, with $55 \%(n=341)$ being done as day cases. None of the patients were positive for COVID-19 post-operatively using reverse transcriptase polymerase chain reaction testing.

Conclusions The risk of COVID-19 following elective cancer surgery in this group of high-risk patients appears to be minimal in this study. With further precautions being introduced to reduce the risk of transmission of COVID-19, an increase in the rate of elective cancer surgery should be a current priority for all hospitals where possible.
\end{abstract}

\section{Introduction}

The COVID-19 pandemic was declared a global health emergency by the World Health Organisation (WHO) on 31 January 2020 [1], and currently 3986119 cases of COVID-19 have been reported worldwide [2]. The overall mortality risk from COVID-19 has been challenging to assess, but the most recent estimation from WHO was

Alison Zoe Luther

alison.luther@porthosp.nhs.uk

1 Department of Surgery, Queen Alexandra Hospital, Portsmouth, UK

2 Department of Breast Surgery, Queen Alexandra Hospital, Cosham PO6 3LY, UK
3.4\% [3-6]. Death rates however have been shown to be much higher in the elderly population, as well as in individuals with comorbidities, or those undergoing treatments resulting in immunosuppression [5, 7-9]. Transmission of COVID-19 is primarily via large droplet spread, and therefore, many countries have instituted major public health strategies including nationwide "lockdowns" and social distancing as an attempt to reduce infection rates $[6,10,11]$.

All health services have made significant changes to face the challenges of COVID-19, aiming to both equip institutions to manage a surge of critically ill patients, and also to minimise face to face contact where possible to reduce further transmission. This has resulted in a substantial reduction in surgical caseload, with most non- 
urgent, non-cancer procedures being suspended in order to reallocate both staff and resources [12-15]. Urgent elective cancer surgery is still proceeding if possible, on an individualised basis according to clinical need, although in some severely affected units, all non-emergency surgery has ceased completely $[13,15,16]$. Some centres have relocated or centralised their elective cancer surgery to hospitals dedicated to planned admissions, aiming to shield patients from potential COVID-19 infection, although this has not been logistically possible for many. As rates of COVID-19 however hopefully begin to decline, enabling cancer surgery to return to full capacity will be a priority for all hospitals, but ensuring the ongoing safety of this patient group remains critical.

Patients with an underlying malignancy have been identified as being in a "high risk" group with regard to COVID-19 and have been strongly advised to implement social distancing [17-19]. Early studies from both China and Italy report poor outcomes in this group of patients, demonstrating that patients with cancer are twice as likely to contract COVID-19, and have increased mortality rates when compared with the general population [6]. This could be due to immunosuppression following neoadjuvant therapies, which may be subsequently compounded by the stress response after surgery [7, 17]. Respiratory function can also be compromised post-operatively, both from the type of surgery performed and also from basal atelectasis following intubation and ventilation. Therefore, careful consideration needs to be given when balancing the risk of cancer progression in the absence of surgical intervention, versus the increased risk of exposure to COVID-19 in this high-risk group of patients. The aim of this paper was therefore to investigate the incidence of COVID-19 in patients following elective cancer surgery in a busy district general hospital and to identify whether risk-reducing precautions were effective.

\section{Methods}

Data was collected from a single large UK-based district general hospital (1200 beds), which was accepting acute admissions with COVID-19 during the study period. The community prevalence of COVID-19 in the region at this time was 196.7 cases per 100,000 of the population [20]. Patients were included in the study if they underwent elective therapeutic cancer surgery between 01/02/20 and $27 / 04 / 20$ and were followed up for a minimum of 30 days post-discharge. All patients scheduled for elective cancer surgery during this period were prioritised clinically using a multi-disciplinary approach.

The initial community public health policies introduced during the study period focused on social distancing, by working from home and staying at home as much as possible. Self-isolation was advised if an individual or household member were symptomatic. A national lockdown was implemented from the 23 March 2020, with the closure of all schools, non-essential shops and hospitality venues. People were only permitted to leave their residences for essential shopping, medical attention, and one hour of exercise per day. The mantra "Stay at home, protect the NHS, save lives", was highly publicised, via governmental and media channels [21, 22].

Within the hospital, multiple precautions were taken to minimise the risk of transmission amongst both staff and patients. Strict increased handwashing and social distancing protocols were implemented where possible. The study hospital has multiple links to the military, including some of the senior leadership team. Awareness of the importance of personal protective equipment (PPE) was therefore paramount, and a highly efficient approach therefore was taken with regard to both centralising PPE and subsequently distributing to it to areas as required. This ensured that there was effective stock control of PPE and good provision to staff throughout the pandemic. Strict adherence to COVID-19 PPE precautions was maintained as advised by the Royal College of Surgeons [15, 23, 24] (RCS) and Public Health England (PHE) [23]. Surgical facemasks were worn by all staff in clinical areas and FFP3 masks were worn when undertaking aerosol generating procedures. A new staff rota was introduced on 30th March 2020, where medical teams were split into working for geographical "zones" rather than specialities, thereby minimising cross-contamination between staff and wards. In the surgical department, operating became consultantled only with only essential staff permitted to enter the operating theatre. Visitors were restricted from entering the hospital, unless visiting patients who were end of life, giving birth or accompanying a minor.

Patients were only tested for COVID-19 using a reverse transcription polymerase chain reaction (RT-PCR) test if symptomatic. Staff were not routinely screened for COVID-19 using RT-PCR testing. A total of 785 symptomatic staff were tested during the study period, with a positivity rate of $22.5 \%$. Acute patients continued to be admitted to the general surgical inpatient wards during this time; however, patients who had suspected COVID-19, or were positive for COVID-19 using RT-PCR testing were barrier nursed in single bedded rooms. Patients who were seen in outpatient clinics had their temperatures screened prior to their appointment.

Patients were initially identified for inclusion using an electronic theatre database and electronic patient records were reviewed retrospectively to collect further information. Data was recorded and analysed using Microsoft Excel. The primary outcome was the incidence of COVID- 
19 in patients during the follow-up period, with COVID-19 positivity being determined by a positive COVID-19 RTPCR test. Secondary outcomes included the number of negative COVID-19 RT-PCR tests, readmissions rates and post-operative mortality during the study period.

\section{Results}

A total of 621 elective cancer surgeries were performed during the 12-week study period. The mean age of the cohort was 63.5 years (range 28-91 years). A wide range of surgical specialities were included, and the breakdown of the procedures by specialty is shown in Fig. 1. 55\% ( $n=341$ ) of procedures were performed as a day case, and the overall mean inpatient stay was 2.36 days (range 1-37 days). During the study period, 859 patients were admitted acutely to the hospital and tested positive for COVID-19 on RT-PCR testing.

$6.4 \%(n=40)$ of the cohort were tested for COVID-19 during the study period, but none of these tested positive for COVID-19 using RT-PCR testing. 3.7\% $(n=23)$ of patients were readmitted post-operatively during the follow-up period; however, none of the readmissions were related to COVID-19 (Table 1$) .0 .8 \%(n=5)$ patients died during the follow-up period. One patient was in hospital and died from acute renal failure. The other four patients were either in their own home, or residential home. Two of these patients died from progression of their underlying malignancy, and it was not possible to elicit the cause of death of the other two patients from the electronic records.

\section{Discussion}

Postponement of elective cancer surgery has huge implications for both patients and healthcare providers; however, this must be balanced against the risk of exposing vulnerable patients to COVID-19 [15]. This study has shown however that by following standard precautions as set out by PHE and RCS, elective surgery for cancer patients can be safely performed in acute hospitals in the current pandemic climate. Interestingly, the patients who required a post-operative inpatient admission did not have increased rates of COVID-19. This is an important finding, which supports increasing the current operative capacity for elective cancer surgery, especially in regions where COVID-19 rates are beginning to decline. It also suggests that redistribution of services to non-acute hospitals is not required to minimise infection rates in this high-risk group of patients, although may be necessary to manage the demand for resources in the local area. The study results are also reassuring for patients awaiting surgery and may help reduce some of the massive psychological stress caused by having a cancer diagnosis in the era of COVID19.

Whilst the study has demonstrated that the risk of COVID-19 is low following cancer surgery, it is important to highlight the need for a thorough, individualised multidisciplinary decision on the timing of surgery. [16] The risks and benefits of each procedure should be carefully weighed against the potential further spread of COVID-19 and the need to utilise our resources in the most effective manner [25]. This will require flexibility at a regional level, given each centre's available resources and the current prevalence of COVID-19 in the local area [13]. It is critical however that units begin to implement strategies to allow elective cancer surgery to be safely reintroduced at the
Fig. 1 Number of procedures performed by specialty

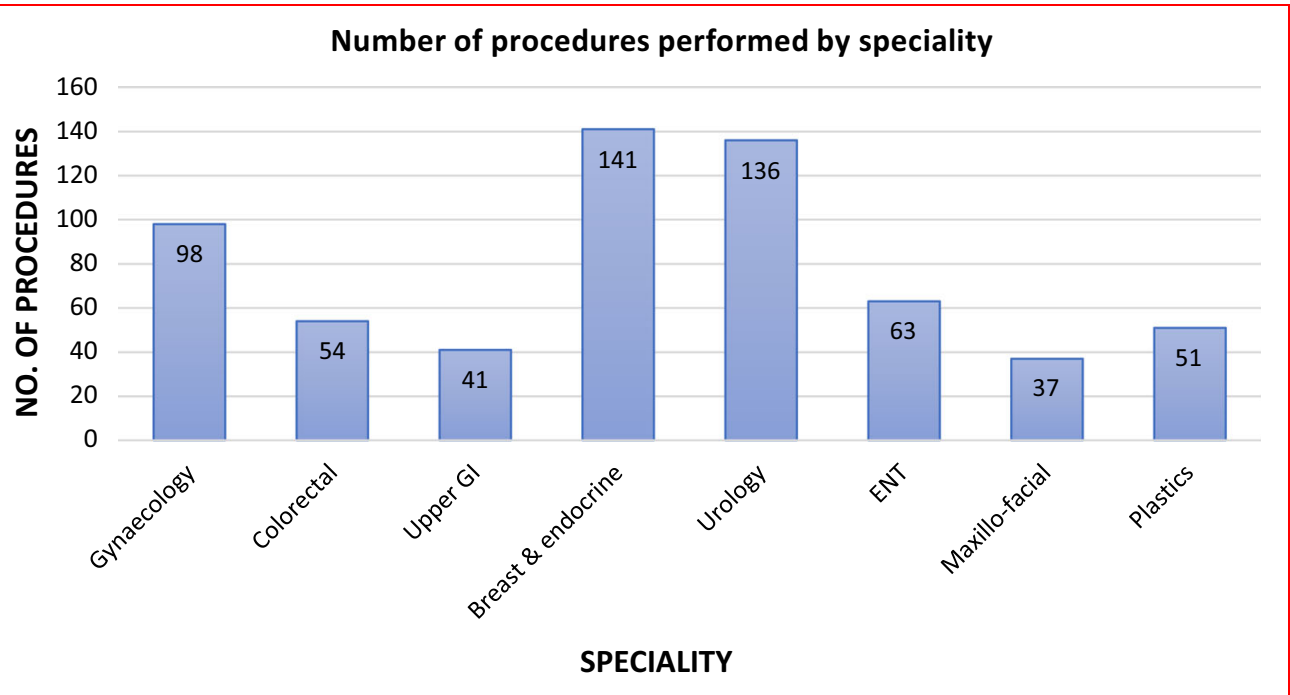


Table 1 Reasons for readmission

\begin{tabular}{lc}
\hline Reasons for readmission & Number of patients \\
\hline Wound infection/intra-abdominal infection & 9 \\
Chemotherapy-related issues & 4 \\
Infection distant from site of operation (not respiratory) & 2 \\
Issues with stoma/enteral feed & 3 \\
Other & 5 \\
\hline
\end{tabular}

earliest point possible, to prevent unnecessary delays in patient care. Careful screening of patients is likely to play an important role in this, and the capacity for testing needs to be developed to allow this to occur [26]. Identifying wards or areas in the hospital which will deal with planned patients only will also be valuable, to prevent transmission of COVID-19 during an inpatient stay. Guidelines for the management of patients undergoing elective surgery are currently being developed to support units in their planning, for example the RCS now recommend that prior to elective surgery patients should be asymptomatic, have self-isolated for fourteen days, and have a negative COVID-19 RT-PCR test, and this has been implemented successfully in the study unit [27]. Testing has become more widely available, and we are now routinely testing patients on admission and pre-operatively. Considerations are also being taken to test all staff members periodically, although this is not currently yet in place. These new developments should serve to maintain the low risk we have demonstrated in our study.

The limitations of this study include a reliance on COVID-19 RT-PCR testing to identify post-operative patients with the disease. Studies have shown testing to have a sensitivity of $80-90 \%$, and therefore, false negatives are to be expected [28, 29]. Clinical information however was obtained for all patients who tested negative to ascertain an overall level of suspicion, including whether there was any radiographic evidence of active COVID-19. This was not evident in any of our patients, and no patients were readmitted for COVID-19 type symptoms post-operatively. During the study period, access to testing was also restricted to patients presenting to hospitals, and therefore, mild cases of COVID-19 would not have been detected, and therefore, the true rate of infection may be higher. It is still important to highlight however that there were no COVID-19 related intensive care admissions or deaths in this group of patients. Another limitation to consider is that clinicians may have selected the fittest cancer patients to operate on and delayed surgery in those with other comorbidities. The patient group in the study may therefore not be representative of the normal population that undergoes elective cancer surgery, and therefore, the risk of COVID-19 may be higher than detected in this study. This highlights the importance for units to continue to develop their pre-operative self-isolation and screening guidelines, and also to identify areas where patients can be managed post-operatively. In the study unit, pre-operative self-isolation and screening have now been implemented successfully, with elective cancer patients being cared for post-operatively on a dedicated ward.

The impact of COVID-19 on elective cancer surgery remains unclear, but it is likely to be significant. Currently, there are concerns regarding a growing backlog of patients who have either had their diagnostic investigations or treatment deferred $[11,13]$, due to a perceived increase risk from COVID-19, and this issue will only worsen if elective cancer surgery rates do not increase. Although this patient group has been previously identified as high risk from COVID-19, the study's findings imply that elective cancer surgery can be performed safely during the current pandemic, using standard precautions only, in an acute hospital managing COVID-19 concurrently. With more stringent pre-operative measures being currently introduced, which will reduce any risk further, it is critical for elective cancer surgery services to resume normal levels of functioning swiftly to prevent further unnecessary patient harm $[13,16,27]$.

\section{References}

1. Landman A, Feetham L, Stuckey D (2020) Cancer patients in SARS-CoV-2 infection: a nationwide analysis in China. Lancet Oncol 21:335-337

2. Xia Y, Jin R, Zhao J et al (2020) Risk of COVID-19 for patients with cancer. Lancet Oncol 21:e180

3. Kutikov A et al (2020) A war on two fronts: cancer care in the time of COVID-19. Ann Intern Med. https://doi.org/10.7326/ m20-1133

4. Diaz A, Sarac BA, Schoenbrunner AR, Janis JE, Pawlik TM (2020) Elective surgery in the time of COVID-19. Am J Surg. https://doi.org/10.1016/j.amjsurg.2020.04.014

5. Liang $\mathrm{W}$ et al (2020) Cancer patients in SARS-CoV-2 infection: a nationwide analysis in China. Lancet Oncol 21:335-337 
6. Spinelli A, Pellino G (2020) COVID-19 pandemic: perspectives on an unfolding crisis. Br J Surg. https://doi.org/10.1002/bjs. 11627

7. Coronavirus (COVID-19) events as they happen. https://www. who.int/emergencies/diseases/novel-coronavirus-2019/events-asthey-happen. Accessed 10 May 2020

8. Coronavirus mortality rate (COVID-19)—worldometer. https:// www.worldometers.info/coronavirus/coronavirus-death-rate/. Accessed 10 May 2020

9. Chen $\mathrm{N}$ et al (2020) Epidemiological and clinical characteristics of 99 cases of 2019 novel coronavirus pneumonia in Wuhan, China: a descriptive study. Lancet 395:507-513

10. Mahase E (2020) Covid-19: death rate is $0.66 \%$ and increases with age, study estimates. BMJ 369:m1327

11. Verity R et al (2020) Estimates of the severity of coronavirus disease 2019: a model-based analysis. Lancet Infect Dis 20(6):669-677

12. Yu J, Ouyang W, Chua MLK, Xie C (2020) SARS-CoV-2 transmission in patients with cancer at a tertiary care hospital in Wuhan, China. JAMA Oncol 1:2. https://doi.org/10.1001/ jamaoncol.2020.0980

13. Bi Q et al (2020) Epidemiology and transmission of COVID-19 in 391 cases and 1286 of their close contacts in Shenzhen, China: a retrospective cohort study. Lancet Infect Dis 20(8):911-919

14. Bedford $\mathbf{J}$ et al (2020) COVID-19: towards controlling of a pandemic. The Lancet 395:1015-1018

15. Iacobucci G (2020) Covid-19: all non-urgent elective surgery is suspended for at least three months in England. BMJ 368:m1106

16. COVID-19: good practice for surgeons and surgical teamsRoyal College of Surgeons. https://www.rcseng.ac.uk/standardsand-research/standards-and-guidance/good-practice-guides/cor onavirus/covid-19-good-practice-for-surgeons-and-surgicalteams/. Accessed 10 May 2020

17. NHS England. Specialty guides for patient management during the coronavirus pandemic. https://www.england.nhs.uk/cor onavirus/wp-content/uploads/sites/52/2020/03/specialty. Accessed 10 May 2020

18. Qadan M, Hong TS, Tanabe KK et al (2020) Multidisciplinary team approach for triage of elective cancer surgery at the Massachusetts general hospital during the novel coronavirus COVID19 outbreak. Ann Surg 1:2020. https://doi.org/10.1097/sla. 0000000000003963

19. Al-Shamsi HO et al. (2020) A practical approach to the management of cancer patients during the novel coronavirus disease 2019 (COVID-19) pandemic: an international collaborative group. The Oncologist. 2020-0213 (2020) https://doi.org/10. 1634/theoncologist.2020-0213. Accessed 10 May 2020

20. Coronavirus cases in the UK: daily updated statistics-GOV.UK. https://www.gov.uk/guidance/coronavirus-covid-19-informationfor-the-public. Accessed 1 June 2020

21. Coronavirus (COVID-19) in the UK - GOV.UK. https://www. gov.uk/government/organisations/public-health-england. Accessed 1 June 2020
22. Our plan to rebuild: The UK Government's COVID-19 recovery strategy - GOV.UK. https://www.gov.uk/government/publica tions/our-plan-to-rebuild-the-uk-governments-covid-19-recov ery-strategy. Accessed 1 June 2020

23. NHS England. Coronavirus » guidance on supply and use of Personal Protective Equipment (PPE) and other supplies. https:// www.england.nhs.uk/coronavirus/publication/guidance-supplyuse-of-ppe/. Accessed 10 May 2020

24. COVID-19: good practice for surgeons and surgical teams Royal College of Surgeons. https://www.rcseng.ac.uk/standardsand-research/standards-and-guidance/good-practice-guides/cor onavirus/covid-19-good-practice-for-surgeons-and-surgicalteams/. Accessed 10 May 2020

25. Ozturk CN, Kuruoglu D, Ozturk C et al (2020) Plastic surgery and the COVID-19 pandemic. Ann Plast Surg. https://doi.org/10. 1097/SAP.0000000000002443

26. Gilbert D (2020) How many coronavirus tests are being done in the UK? May 19, 2020. https://www.telegraph.co.uk/news/0/cor onavirus-testing-how-many-tests-done-uk/ Accessed 20 May 2020

27. Recovery of surgical services during and after COVID-19 Royal College of Surgeons. https://www.rcseng.ac.uk/cor onavirus/recovery-of-surgical-services/. Accessed 10 May 2020

28. Long $\mathrm{C}$ et al (2020) Diagnosis of the coronavirus disease (COVID-19): rRT-PCR or CT? Eur J Radiol 126:108961

29. Grassly N (2020) Report 16-role of testing in COVID-19 control I Faculty of Medicine I Imperial College London. https:// www.imperial.ac.uk/mrc-global-infectious-disease-analysis/ covid-19/report-16-testing/. Accessed 10 May 2020

Publisher's Note Springer Nature remains neutral with regard to jurisdictional claims in published maps and institutional affiliations.

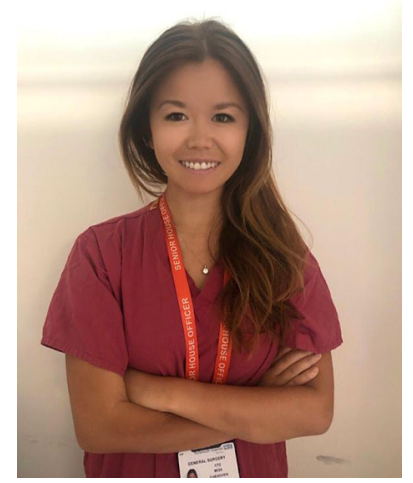

Chenchen Ji graduated with a MBBS BSc from Imperial College, London, UK, in 2015. She is currently working as a core surgical trainee at Queen Alexandra Hospital and has recently accepted a higher surgical training post in the Wessex deanery. She obtained membership to the Royal College of Surgeons (Eng) in 2017 and is looking forward to developing a career in general surgery. 